\title{
A Superconducting Magnetic Lens for Solar Rays Protection in Manned Interplanetary Missions
}

\author{
Lucio Rossi, Massimo Sorbi, and Piero Spillantini
}

\begin{abstract}
During the interplanetary flights the crewmembers will be exposed to cosmic ray radiation (CR) with great risk for their health. The adsorbed dose due to $C R$ depends on the galactic (GCR) or solar (SCR) origin. GCRs are isotropic and relatively high in energy and deliver a dose nearly constant. The SCRs are usually much less energetic, of the order of few tens of $\mathrm{MeV}$ but during some exceptional solar bursts, a great number of particles, mainly protons, are ejected at higher energies. In this case the dose delivered in a few hours by a solar burst inside a spacecraft can easily exceed 1 year cumulated dose by GCRs. The high-energy component of SCRs is quasidirectional so that a shielding system based on a superconducting magnetic lens (a toroid) can reduce the dose rate of SCRs to the level delivered by GCRs. This paper presents the concept, the general aspects and the main technical solutions that are adopted for this magnet.
\end{abstract}

Index Terms-Superconducting coils, superconducting magnets in the space, superconducting toroids.

\section{INTRODUCTION}

$\mathbf{T}$ HE HUMAN exploration of the Solar System by manned missions will be possible only when the problem of the protection of the astronauts from the cosmic ionizing radiation will be solved.

In particular, very dangerous is the solar cosmic ray component (SCR), emitted in violent solar events. The most large and energetic of these solar bursts generate a very high effluence of particles (mainly protons) with an energetic tail. The total dose could result lethal for the astronauts and, because of the high energy components $\left(E_{\text {proton }}>100 \mathrm{MeV}\right)$, it is not obvious to stop it in absorbing materials because the required mass is of the order of several tonnes, with adverse effects on the useful load of the cargo.

A large fraction of these energetic particles is coming from a given direction with a few degrees angular divergence, and therefore, they can be deviated by a relatively compact superconducting magnetic lens. We know from previous evaluations that one single lens can protect from this mono-directional component of the radiation flux, a volume as large as those of an ISS-like shielded module (International Space Station), i.e., a cylinder of about $2 \mathrm{~m}$ diameter and some $\mathrm{m}$ of length [1], [2].

This work was finalized to assess what could be the difficulties for constructing in a few years, based on present available and reliable techniques, such a lens and what would be its final

Manuscript received October 20, 2003.

L. Rossi is with CERN-Accelerator Technology division on leave from Milan University-Physics Department (email: lucio.rossi@cern.ch).

M. Sorbi and P. Spillantini are with Physics Department, Firenze University, 50019 Sesto Fiorentino (FI) Italy (e-mail: massimo.sorbi@mi.infn.it; spillantini@ fi.infn.it).

Digital Object Identifier 10.1109/TASC.2004.831041

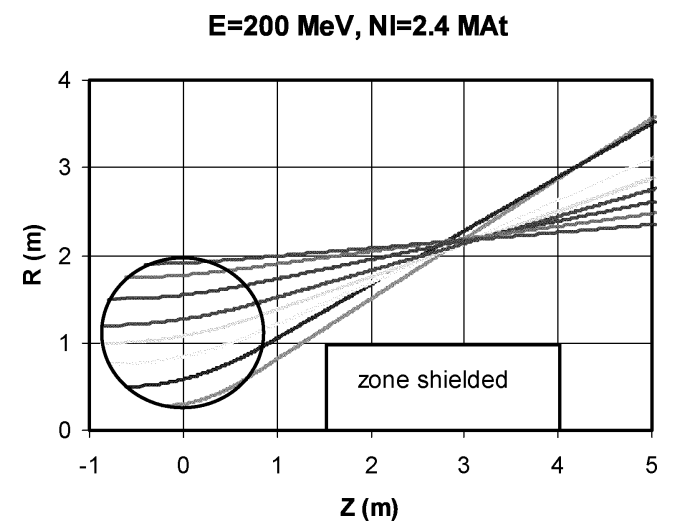

Fig. 1. Trajectories of monodirectional proton in toroidal field.

parameters, in particular its mechanical structure, its mass and its power consumption.

For the design we assumed that the particles arrive with an angular divergence of $+/-10$ degrees. We design the lens capable to clear up the entire volume of $\varnothing 2 \mathrm{~m} \times \mathrm{L} 2.5 \mathrm{~m}$ from protons up to $200 \mathrm{MeV}$, while accepting a decrease of the protection efficiency for higher energy protons.

It is necessary to underline that until now there are not sufficient data for a definitive assessment of how big is the SCR component that can be considered as directional, although data given by support the directionality as the main component. This important information will be available only in the future. If it would result that at energies exceeding about $50 \mathrm{MeV}$ the nondirectional fraction of the SCR is large, it will be necessary understand what magnetic configuration could be assumed for protecting the astronauts from energetic particles arriving from all the directions.

\section{IDEAL TOROID: CirCUlaR COIL CONFIGURATION}

Some preliminary studies have demonstrated that a toroidal field is very efficient for the deflection of energetic protons coming from a direction; infact, if the toroid axis is parallel to the direction of the incoming protons, the deflection force is always effective, because the magnetic field is perpendicular to the protons trajectories. The field inside the toroid decreases vs. the increasing of the distance from the toroid axis $(\mathrm{B} \div 1 / \mathrm{R})$; consequently the protons at lower radii are more deflected than the protons at higher radii as required by the shape of the protection volume (see Fig. 1). The magnet is designed to be wound with classical low- $T_{c}$ superconductors and to be cryogen-free in order to avoid to depend on liquid helium which might be lost, particularly following a quench and is in any case a source of concern for the safety of the mission. 
TABLE I

MAIN PARAMETES OF TOROID WITH Circular Cross SECTION

\begin{tabular}{cc}
\hline \hline Parameter & Value \\
$\mathrm{R}_{\min }$ & $0.25 \mathrm{~m}$ \\
$\mathrm{R}_{\max }$ & $2.0 \mathrm{~m}$ \\
$\rho=\left(\mathrm{R}_{\max }-\mathrm{R}_{\min }\right) / 2$ & $0.875 \mathrm{~m}$ \\
$\mathrm{NI}$ & $2.4 \mathrm{E} 6 \mathrm{At}$ \\
$\mathrm{B}_{\max }$ & $1.92 \mathrm{~T}$ \\
\hline \hline
\end{tabular}

The shape of the coil should be optimized in order to maximize the deflection efficiency, to minimize the mass of conductor and to minimize the stress on the coil. As preliminary study, the approximation of perfect toroidal field has been used and the field is supposed to be produced by a uniform distribution of current with toroidal symmetry. In this way the comparison between different solutions is easier because the field can be calculated analytically. Thereafter the advantage of using a finite number of coils (lumped toroid) over the uniform current distribution will be assessed.

In previous studies [1], [2], toroidal field produced by circular coils had been considered. The Table I reports the main parameter of this type of toroid, and Fig. 1 gives a representation of the trajectories of protons with the action of the toroidal field. This type of configuration is able to protect a cylinder of radius $1 \mathrm{~m}$ and with a length between $1.5 \mathrm{~m}$ and $4.0 \mathrm{~m}$ from the center of the toroid ( $\mathrm{Z}$ axis in Fig. 1). The zone with $\mathrm{R}<\mathrm{R}_{\min }$ is assumed to be protected by passive absorber positioned in the central hole of the toroid. Actually this zone can be best used to allocated electrons detectors. Electrons reaches the space ship some 5 to 15 minutes in advance and indicates the incoming directions of the protons, allowing an adjustment of the toroid axis to maximize the protection efficiency.

This coil configuration is simple to build and it makes the winding easy. On the other hand it requires a mechanical structure to withstand the considerable e.m. force that tends to deform the circular shape of the coil, with a considerable increasing of the total mass.

\section{IDEAL TOROID: THE "D” ShAPED CONFIGURATION}

The analysis of the stresses and deformation becomes much easier if the configuration that keeps constant the tension of the conductor for the magnetic forces is adopted. In this case the radius of curvature of the turn $\rho$ cannot be constant but has to satisfy the condition [3]:

$$
T=B \cdot N I \cdot \rho=\text { costant } .
$$

In this case all the magnetic pressure can be contained by the sole tension of the conductor and the coil cross section assumes a "D" shape. Moreover, the " $D$ " shaped coils have the great vantage to increase the deflection efficiency in the zone close to $R_{\text {min }}$ : infact the length of the coils is much larger for low radii than for large radii. The choice of $R_{\max }$ is given by the condition to intercept the trajectories of the protons in the zone that has to be protected. If we assume that the angular distribution of the proton trajectories is $\pm 10^{\circ}, R_{\max }$ results equal to $1.71 \mathrm{~m}$. In this case the length of the coils increase greatly respect to the case with circular coils, and it is possible to reduce drasti-
$E=200 \mathrm{MeV}, \mathrm{Nl}=1.0 \mathrm{MAt}$

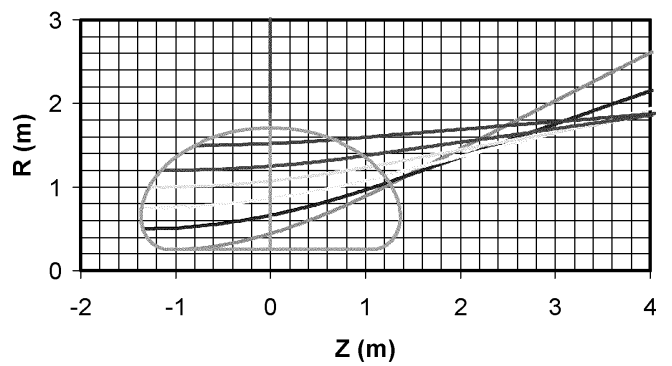

Fig. 2. Trajectories of monodirectional protons in toroidal field with coils "D" shaped, with $R_{\max }=1.71 \mathrm{~m}$.

cally the total current of the in the magnet. The Fig. 2 shows the trajectories of monodirectional protons inside the toroid with $R_{\max }=1.71 \mathrm{~m}$ and $N I=1.0 \mathrm{E} 6 \mathrm{At}$.

The integral tension on the conductor is $0.19 \mathrm{MN}$. If we assume that the current density in the overall conductor is 100 $\mathrm{A} / \mathrm{mm}^{2}$, the stress on the conductor is:

$$
\sigma=\frac{T_{\max }}{S_{\text {cond }}}=T_{\max } \frac{J_{\text {overall }}}{N I} \approx 19.0 \mathrm{MPa},
$$

which can be accepted by any superconductor in $\mathrm{NbTi}$ or $\mathrm{Nb}_{3} \mathrm{Sn}$.

\section{THE "D" SHAPE FOR A LUMPED TOROID}

Ideal toroids have further advantages over lumped coil configuration:

1) the field is uniform in azimuth, being axis-symmetric;

2) therefore the field outside of the toroid vanishes.

No stray field is really an advantage in space environment, since elimination of any interference with all activities that can be sensitive to the field lessens the concerns about safety. On the other hand an ideal toroidal field requires a continuum of winding, which is not at all practical as all configuration requiring strong 3-D coils. Moreover a distributed coil shows a large surface to be protected from thermal radiations, and a large cooling power would be required. In order to make more practical and less expensive the construction and to reduce the surface exposed to radiation power, the configuration of magnetic field produced by a discrete and limited number of coils has been considered. Also in this case, the "D" shape makes the e.m. forces fully supported by the tension of the conductor. Again the curvature radius $\rho$ of the coils has to be chosen in order to fulfill the condition (1). In this case, the magnetic field on the conductor doesn't vary anymore like 1/R, like it does in an ideal toroid: it can be estimated that the field profile on the conductor is described by the relation:

$$
B(R)=B_{0} \frac{R_{0}^{\alpha}}{R^{\alpha}}
$$

where the exponent $\alpha$ is a number between 0 and 1 that can be found with a best fit of the calculated values of $B$. With this assumption, the ideal shape of the coil in the plane Z-R can be determined [4].

As numerical example, the configuration of toroid with 4 coils has been considered. The Fig. 3 reports the calculated value 


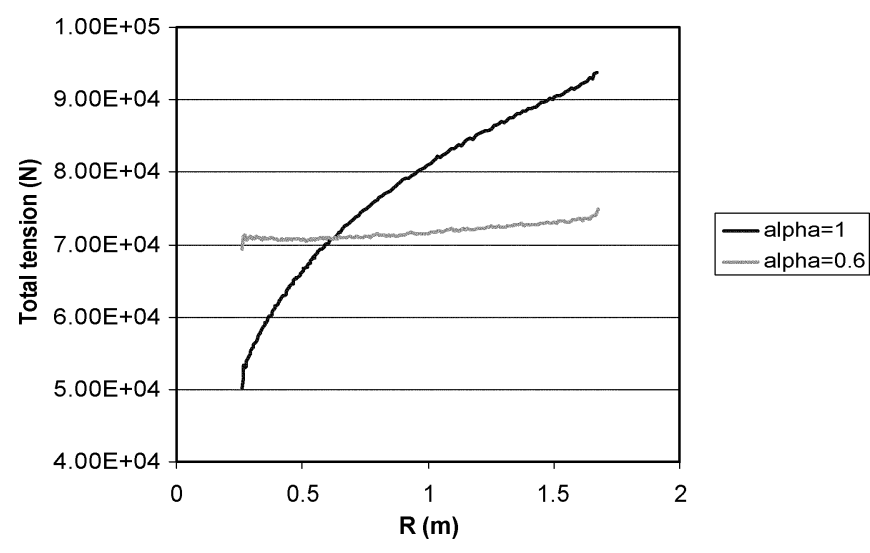

Fig. 3. Total tension on each coil, with configuration optimized for ideal toroid $($ alpha $=1)$ or for 4 coils $($ alpha $=0.6) . \mathrm{NI}=1.55 \mathrm{MAt}$ in total.

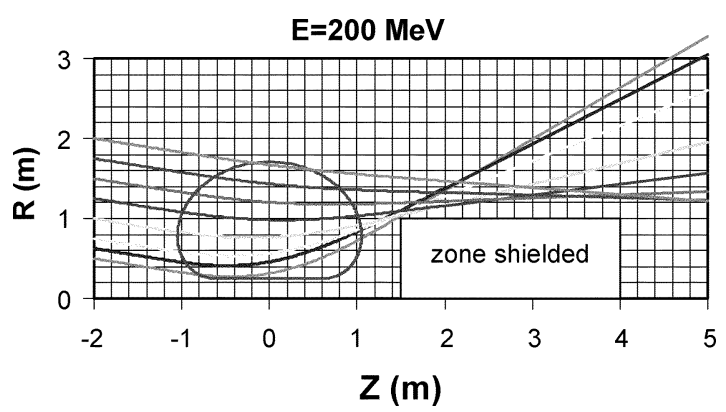

Fig. 4. Trajectories of protons in the plane between two coils, with an initial angle of $-10^{\circ}$. NI $=2.0 \mathrm{MAt}$ in total, and the coil number is 4 . The coil profile is represented as reference.

of the tension of the coils with a final best fit for $\alpha=0.60$. It can be observed that with the optimized configuration for 4 coils $(\alpha=0.6)$ the tension is almost constant, whereas in the configuration of ideal toroid $(\alpha=1)$ the variation of the tensions is large. The variation of the tension on the conductor for $\alpha=0.6$ is limited to less than 5\%, which can be considered sufficient for this kind of study.

The Fig. 4 reports the shape of the coil. As it was expected, the dimension of the coil in the $\mathrm{Z}$ direction is sensibly reduced respect the case of ideal toroid (Fig. 2), because the variation of the field on the coil is lower than in the case of ideal toroid.

Passing to a discrete number of coils, it means that the field is not anymore axis symmetric and some stray field appears, with reduction of the field strength in between the coils.

Fig. 4 represents the protons trajectories in the worst situation, i.e., in the plane at 45 degrees between two coil plane (where the field is lowest) and with the protons coming with an angle of -10 degrees. It is possible to note that with a total current of 2.0 MAt, i.e., about $15 \%$ more than for the correspondent ideal toroid configuration) the zone of interest is completely protected.

\section{CONDUCtor Evaluation}

In the choice of the conductor, the following aspects have to be considered: quantity and type of matrix (stabilization metal) for the protection and stability of the magnet; type of superconductor ( $\mathrm{NbTi}$ vs. $\mathrm{Nb}_{3} \mathrm{Sn}$ ); thickness of electrical insulation of the turns.
TABLE II

Main GeOMETRICAL AND Electrical PaRAMETERs of the TOROID (4 COILS CONFIGURATION)

\begin{tabular}{lc}
\hline \hline Conductor cross section (w/o insulation) & $2 \mathrm{~mm} \times 2 \mathrm{~mm}$ \\
Conductor cross section (with insulation) & $2.5 \mathrm{~mm} \times 2.5 \mathrm{~mm}$ \\
Coil cross section & $157.5 \mathrm{~mm} \times 50 \mathrm{~mm}$ \\
Turn number & $63 \times 20$ \\
Max radius of coil cross section center & $1.71 \mathrm{~m}$ \\
Min radius of coil cross section center & $0.25 \mathrm{~m}$ \\
Average hoop stress on conductor & $23.4 \mathrm{MPa}$ \\
Current & $397 \mathrm{~A}$ \\
Magnetic energy & $2.3 \mathrm{MJ}$ \\
Total conductor mass (w/o insulation) & $280 \mathrm{~kg}$ \\
\hline \hline
\end{tabular}

The matrix types, which usually are used for the stability and protection of low temperature superconductor, are copper or aluminum. Between these two materials, aluminum is preferable, because its specific enthalpy increasing is considerable higher than copper, in a temperature variation from $6 \mathrm{~K}$ to $100 \mathrm{~K}$. Here it is important to underline that in space flight the weight is a fundamental criteria in the design; therefore it is correct to compare the specific enthalpy rather than the volumetric enthalpy, as it is usually done for most of superconducting magnets, where coil volume rather than coil weight is the predominant factor.

Consequently, at constant mass, the aluminum temperature increasing, due to internal dissipation of magnetic energy, is sensibly lower than in the copper case. The stabilizer cross section is determined, in first instance, by the necessity to limit the increasing of temperature of the magnet during a quench. As design choice, we limit the coil temperature increasing to about 80-90 K, assuming that all the magnetic energy is uniformly dissipated inside the coils. In Table II the main geometrical parameters of the coils (4 coils configuration) are reported. With these parameters the temperature increase results below $80 \mathrm{~K}$. The calculation has been performed with-out considering the enthalpy contribution of insulation and assuming that all the metallic part of conductor is aluminum.

In the evaluation of the superconductor type, we decided to take in consideration only $\mathrm{NbTi}$ and $\mathrm{Nb}_{3} \mathrm{Sn}$, i.e., we excluded "a priori" the high temperature superconductors that will be considered in a second phase.

We fix a margin of temperature for the stability (difference between current sharing temperature and operation temperature) of $1.5 \mathrm{~K}$, in order to allow some thermal gradient to evacuate heat to the heat sink (being cryogen free, cooling relies only on conduction). Moreover we assume to work at moderate current density in the superconductor, since high $\mathrm{J}_{\mathrm{Sc}}$ are useless for the weight (dominate by the stabilizer content) and requires too low temperatures. $\mathrm{J}_{\mathrm{Sc}}$ is then fixed at $1500 \mathrm{~A} / \mathrm{mm}^{2}$ for $\mathrm{NbTi}$ and $1000 \mathrm{~A} / \mathrm{mm}^{2}$ for $\mathrm{Nb}_{3} \mathrm{Sn}$; this difference takes in account that $\mathrm{Nb}_{3} \mathrm{Sn}$ is somehow less reliable and much more easy to degrade than $\mathrm{NbTi}$. In order to define the current sharing temperature, it is necessary to know precisely the maximum magnetic field in the superconductor. The calculation of the field has been performed analytically, and the maximum field varies considerable with the coil number. In all the considered configurations, the total current in the magnet is $2.4 \mathrm{MAt}$, except for the Perfect Toroid case, where NI = 1.0 MAt. The results are presented in Table III. 
TABLE III

Peak Field AND Operation Temperature vs. CoIl Number of Magnet

\begin{tabular}{lcccc}
\hline \hline & 3 coils & 4 coils & 5 coils & Perfect toroid \\
\hline Bpeak on supercond. & $3.5 \mathrm{~T}$ & $2.8 \mathrm{~T}$ & $2.4 \mathrm{~T}$ & $0.8 \mathrm{~T}$ \\
Oper. Temp. with NbTi & $5.37 \mathrm{~K}$ & $5.85 \mathrm{~K}$ & $6.11 \mathrm{~K}$ & 7.09 \\
Oper. Temp. with NbSn & $8.01 \mathrm{~K}$ & $8.99 \mathrm{~K}$ & $9.57 \mathrm{~K}$ & $12.17 \mathrm{~K}$ \\
\hline \hline
\end{tabular}

TABLE IV

THERMAL LOAdS vs. COILS Number OF THE MAGNET

\begin{tabular}{lcccc}
\hline \hline & 3 coils & 4 coils & 5 coils & Perfect toroid \\
\hline & \multicolumn{5}{c}{ Thermal load on cold mass } \\
Radiation & $0.145 \mathrm{~W}$ & $0.193 \mathrm{~W}$ & $0.241 \mathrm{~W}$ & $0.473 \mathrm{~W}$ \\
Supports & $0.010 \mathrm{~W}$ & $0.013 \mathrm{~W}$ & $0.016 \mathrm{~W}$ & $0.015 \mathrm{~W}$ \\
Cold mass Total & $0.16 \mathrm{~W}$ & $0.21 \mathrm{~W}$ & $0.26 \mathrm{~W}$ & $0.49 \mathrm{~W}$ \\
\hline \multicolumn{5}{c}{ Thermal load on cooled shield $(70 \mathrm{~K})$} \\
Radiation & $12.3 \mathrm{~W}$ & $15.8 \mathrm{~W}$ & $19.3 \mathrm{~W}$ & $19.2 \mathrm{~W}$ \\
Supports & $0.63 \mathrm{~W}$ & $0.84 \mathrm{~W}$ & $1.05 \mathrm{~W}$ & $1.05 \mathrm{~W}$ \\
Thermal shield Total & $12.9 \mathrm{~W}$ & $16.6 \mathrm{~W}$ & $20.3 \mathrm{~W}$ & $20.3 \mathrm{~W}$ \\
\hline \hline
\end{tabular}

In the configuration with 3,4 or 5 coils, the radius of curvature for conductor is below $285 \mathrm{~mm}$, that corresponds to a relative elongation of $0.35 \%$ with a conductor $2 \mathrm{~mm}$ thick; for this reason, with $\mathrm{Nb}_{3} \mathrm{Sn}$, the React and Wind technique can be adopted, which allows to use aluminum as stabilization (the strand of superconductor can be soldered on the matrix of aluminum after the thermal treatment necessary to obtain $\mathrm{Nb}_{3} \mathrm{Sn}$ [4]) and the problem of insulation is very much simplified.

From this comparative study results that a configuration with greater number is preferable, because the peak field is lower and the operation temperature can be higher. However this point will have to be discussed again in the thermal and mass evaluation. Both $\mathrm{NbTi}$ and $\mathrm{Nb}_{3} \mathrm{Sn}$ can be used as superconductor. The difference in the temperature operation between these two materials is few kelvin, and increases with the number of coils.

\section{Thermal Evaluation}

A detailed description of the thermal calculation for the magnet is described in a different paper [5]. Here we only report that a first external passive radiation shield screens the magnet from the sun radiation; inside this radiation shield there is second shield cooled at $70 \mathrm{~K}$. The total thermal power depends by the number of coils of the magnet, and is reported in Table IV.

The configuration with 3 coils requires about $0.25 \mathrm{~W} @ 5.85$ $\mathrm{K}$ and less than $15 \mathrm{~W} @ 70 \mathrm{~K}$, i.e., figures that translate in less than $1.0 \mathrm{~kW}$ electrical power. The cooling power is well within the reach of many crycooler types and we think that the issues here are mainly about reliability and design adaptation to
TABLE $\mathrm{V}$

Masses of the MAgnet COMPONENTS vs. COIL Number of THE MAGNET

\begin{tabular}{lcccc}
\hline \hline & 3 coils & 4 coils & 5 coils & Perfect toroid \\
\hline Conductor mass & $315 \mathrm{~kg}$ & $414 \mathrm{~kg}$ & $512 \mathrm{~kg}$ & $80 \mathrm{~kg}$ \\
Total cold mass & $380 \mathrm{~kg}$ & $500 \mathrm{~kg}$ & $620 \mathrm{~kg}$ & $598 \mathrm{~kg}$ \\
Thermal shield mass & $103 \mathrm{~kg}$ & $132 \mathrm{~kg}$ & $162 \mathrm{~kg}$ & $195 \mathrm{~kg}$ \\
Radiation shield mass & $223 \mathrm{~kg}$ & $283 \mathrm{~kg}$ & $343 \mathrm{~kg}$ & $320 \mathrm{~kg}$ \\
Suspensions & $8 \mathrm{~kg}$ & $8 \mathrm{~kg}$ & $10 \mathrm{~kg}$ & $20 \mathrm{~kg}$ \\
Total mass & $713 \mathrm{~kg}$ & $922 \mathrm{~kg}$ & $1134 \mathrm{~kg}$ & $1133 \mathrm{~kg}$ \\
\hline \hline
\end{tabular}

the space environment (limitation of the "plug" power and heat rejection at higher temperature).

\section{MASS EVALUATION}

The convenience in adopting an active shield vs. a passive shield form SCR has mainly to be evaluated from the point of view of the total mass of the system. Indeed weight, rather than volume, is the key factor in the payloads. In Table $\mathrm{V}$ the masses of the magnets are reported, for different configurations. In the calculation, the mass of the cryocoolers is not considered but its weight can be estimated in less the $50 \mathrm{~kg}$. As reference, passive absorber has a mass of about $3000 \mathrm{~kg}$, in order to protect the same zone considered in the active shield.

\section{CONCLUSION}

This study demonstrates the feasibility of an active system for the protection from SCRs. The values of magnetic field required and the operation temperatures allow the use of both $\mathrm{NbTi}$ and $\mathrm{NbSn}$ as superconductor, and the temperature range allows the use of a cryocooling system. The configuration with 3 coils is preferable because it allows lower thermal power consumption and has a lower mass, even if the operation temperature is slightly lower respect the other configuration. With this solution the mass of the protection system can be about 4 times lower than the mass of a passive system a figure that make attractive and rewarding to pursue the road of magnetic shielding.

\section{REFERENCES}

[1] P. Spillantini, F. Taccetti, P. Papini, and L. Rossi, "Radiation Shielding of Spacecrafts in Interplanetary Flights,", INFN/AE-99/002, 1999.

[2] - "Radiation shielding of spacecrafts in interplanetary flights," Nuclear Instruments \& Methods in Physics Research, vol. A 443, pp. 254-263, 2000.

[3] M. Wilson, Superconducting Magnets: Claredon Press Oxford, 1983.

[4] P. Turowki and T. Schneider, "19.3 T with a superconducting magnet," IEEE Trans. Magn., vol. 24, no. 2, pp. 1063-1066, March 1988.

[5] L. Imbasciati, L. Rossi, M. Sorbi, and P. Spillantini, Optimization of Superconducting Coils of a Magnetic Shield for Solar Cosmic Rays Protection During Interplanetary Missions. 